\title{
Functional integration of two CYP450 genes involved in biosynthesis of tanshinones for improved diterpenoid production by synthetic biology
}

Yaping $\mathrm{Mao}^{\mathrm{a}, \uparrow}$, Ying $\mathrm{Ma}^{\mathrm{a}, \dagger}$,Tong Chen ${ }^{\mathrm{a}}$, Xiaohui $\mathrm{Ma}^{\mathrm{b}}$, Yanqin $\mathrm{Xu}^{\mathrm{c}}$, Junling $\mathrm{Bu}^{\mathrm{a}}$, Qishuang Li ${ }^{\mathrm{a}, \mathrm{d}}$, Baolong Jin ${ }^{\mathrm{a}}$, Yanan Wang ${ }^{\mathrm{a}}$, Yong Lia ${ }^{\mathrm{a}, \mathrm{e}}$, Guanghong Cui ${ }^{\mathrm{a}}$, Yujun Zhao ${ }^{\mathrm{a}}$, Jinfu Tang ${ }^{\mathrm{a}}$, Ye Shen ${ }^{\mathrm{a}}$, Changjiangsheng Lai ${ }^{\mathrm{a}}$, Wen Zeng ${ }^{\mathrm{a}}$, Min Chen ${ }^{\mathrm{a}}$, Juan Guo ${ }^{\mathrm{a}, *}$, and Luqi Huang a,*

a State Key Laboratory Breeding Base of Dao-di Herbs, National Resource Center for Chinese Materia Medica, China Academy of Chinese Medical Sciences, Beijing 100700, China;

b Yunnan University of Traditional Chinese Medicine,Kunming 650500, China;

${ }^{\mathrm{c}}$ College of Pharmacy, Jiangxi University of Traditional Chinese Medicine, Nanchang 330004, China;

d School of Pharmacy, Jiangsu University, Zhenjiang 212013,China;

e Shandong University of Traditional Chinese Medicine, Jinan 250355, China.

${ }^{\dagger}$ These authors have equally contributed to this manuscript.

* Authors to whom correspondence should be addressed:

E-mail: guojuanzy@163.com; ORCID: 0000-0002-1327-4428 (J.G.)

E-mail: huangluqi01@126.com; ORCID: 0000-0002-2070-4318 (L-Q.H)

Tel.: +86-010-64087469 (J.G.); Fax: +86-010-84027175 (J.G.). 
A

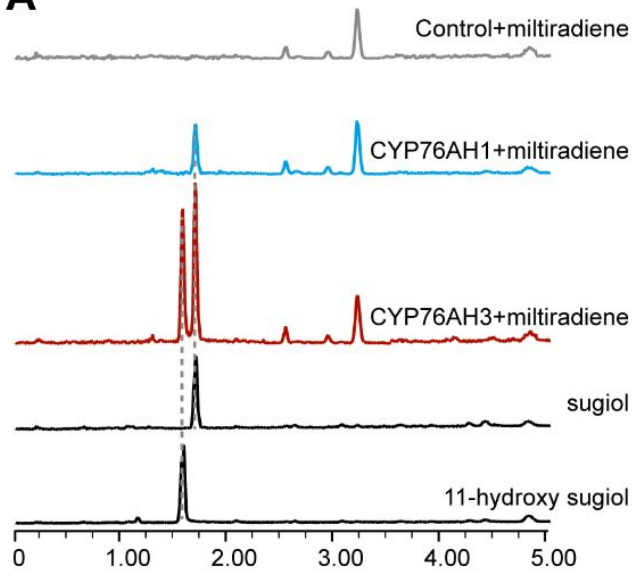

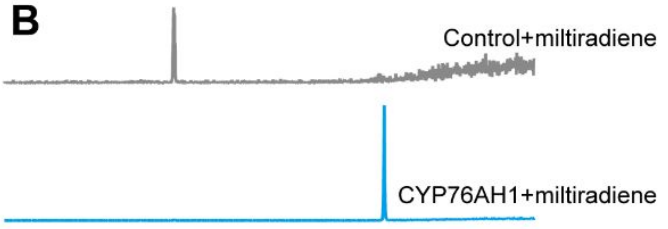

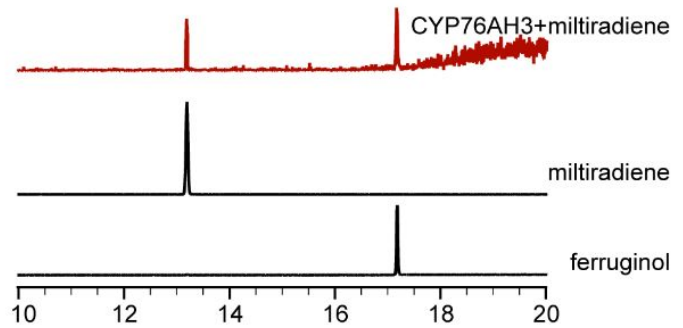

C

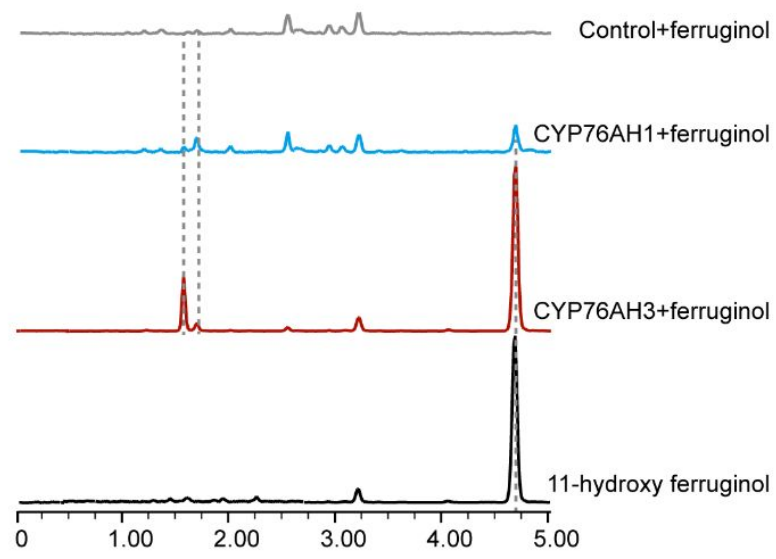

Figure S1. In vitro enzyme assay of CYP76AH1 and CYP76AH3 using miltiradiene $(\mathbf{A}, \mathbf{B})$ and ferruginol $(\mathbf{C})$ as substrate. $\mathbf{A}$ and $\mathbf{C}$ are enzyme reaction analysis in UPLCQtof-MS, B is the GC-MS result of miltiradiene enzyme reaction. 

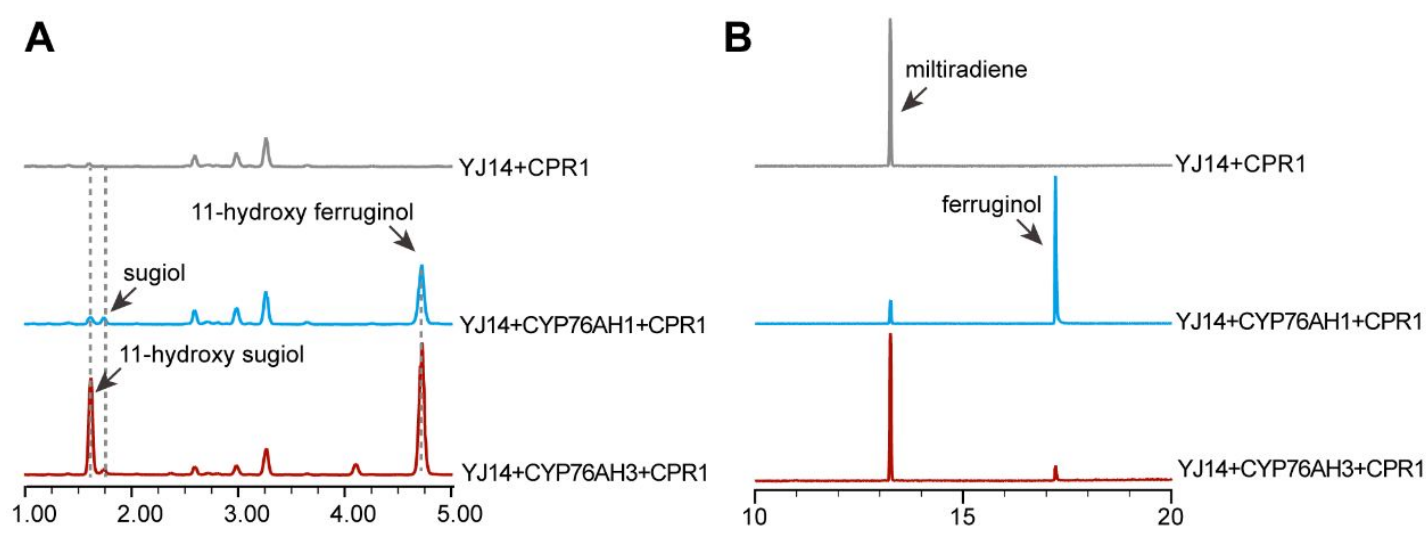

Figure S2. UPLC-Qtof-MS result (A) and GC-MS result (B) of diterpnoid production in the yeast strain harboring miltiradiene production module (YJ14) plus CPR1 from Salvia miltiorrhiza (grey), miltiradiene production module (YJ14) plus CYP76AH1 and CPR1 (blue), and miltiradiene production module (YJ14) plus CYP76AH3 and CPR1 (red). 
A

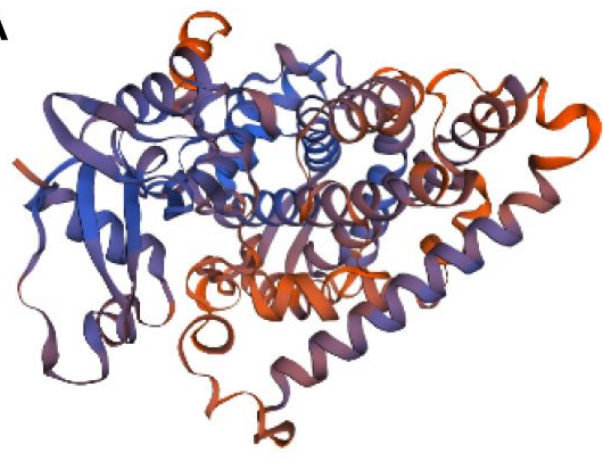

C

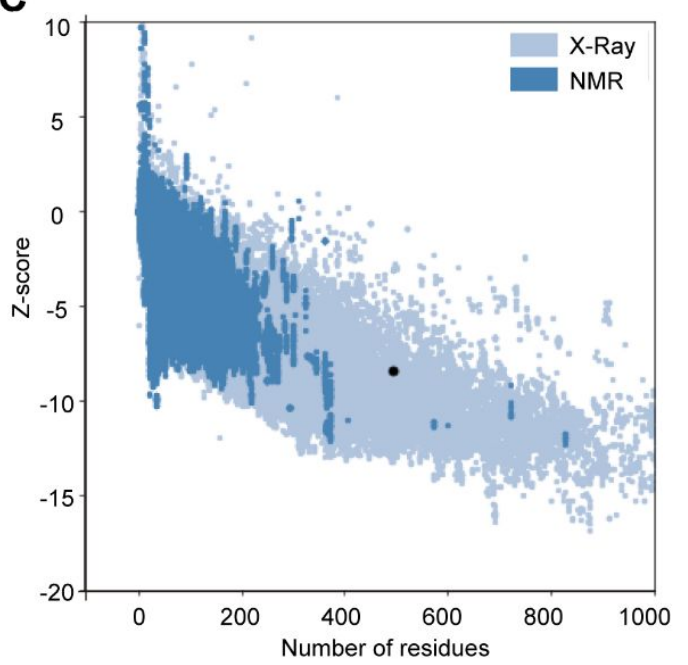

B

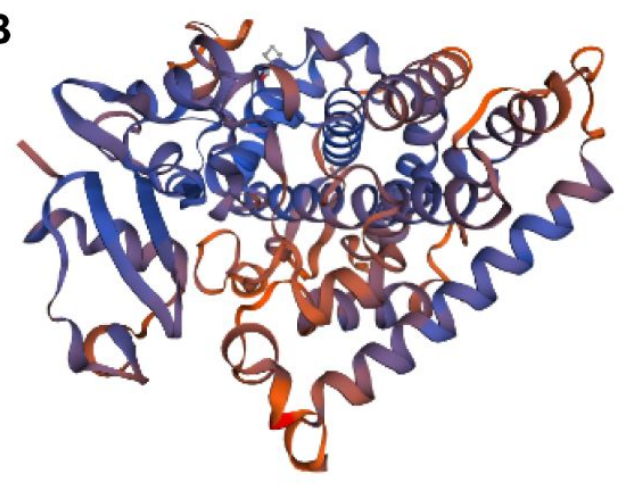

D

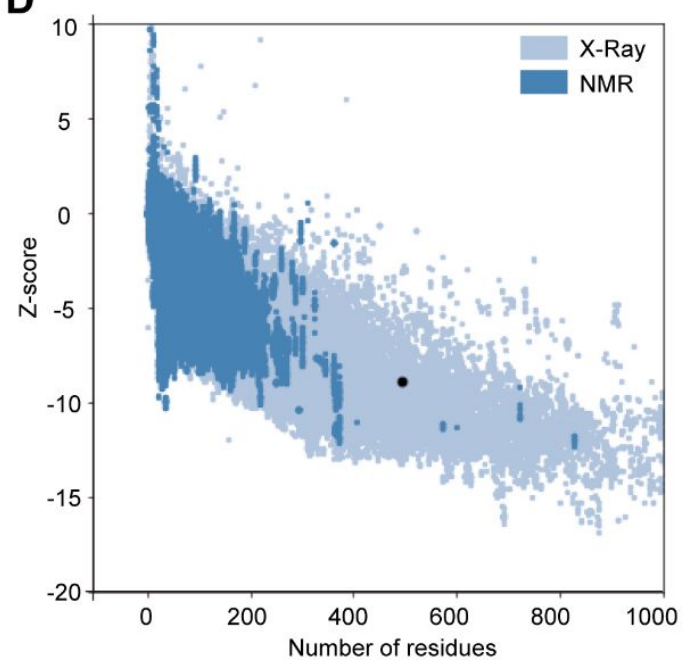

Figure S3. Homology modeling of CYP76AH1 (A) using the crystal structure of CYP1A2 from human as template, and homology modeling of CYP76AH3 (B) using the crystal structure of CYP17A1 from zebra fish as template. The ProSA energy graphs for $\mathrm{CYP76AH1}=-8.4$ with 495 amino acid residues in the model $(\mathbf{C})$ and for CYP76AH3 $=-8.84$ with 494 amino acid residues in the model (D) were all in a negative range indicating the reasonable model for model. 
A

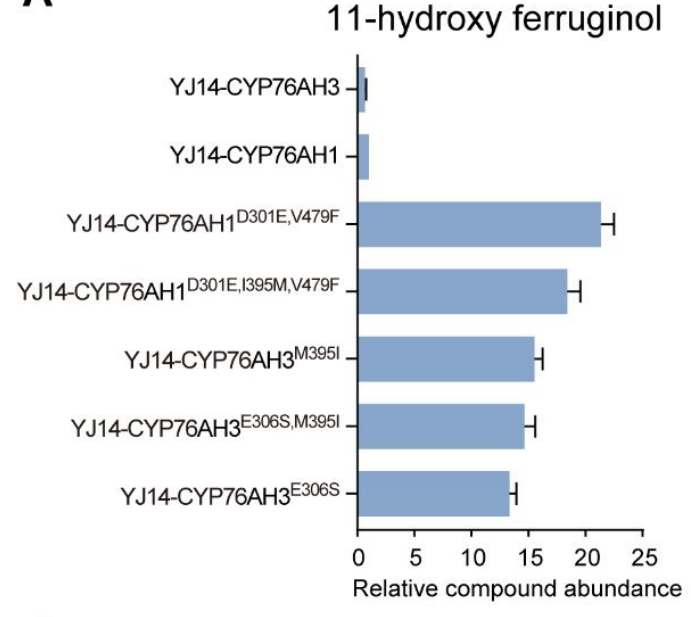

C

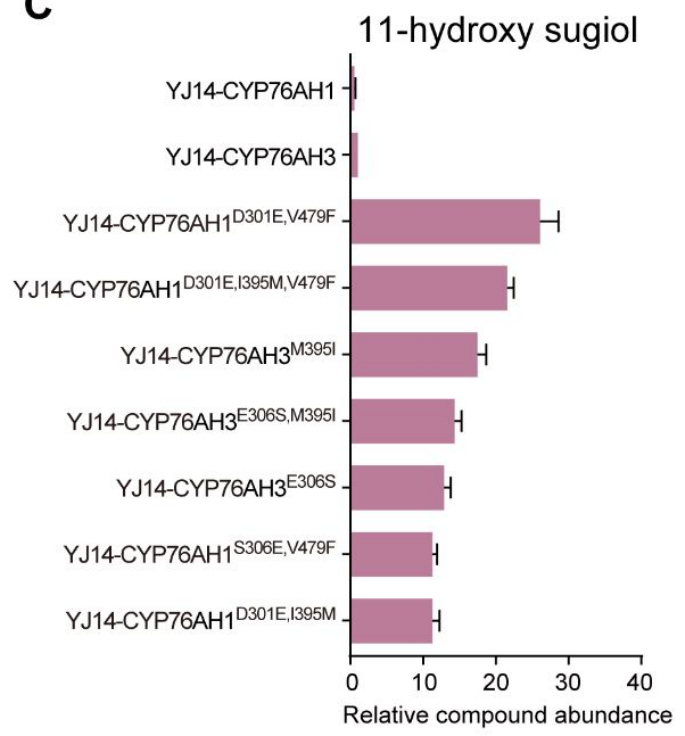

B

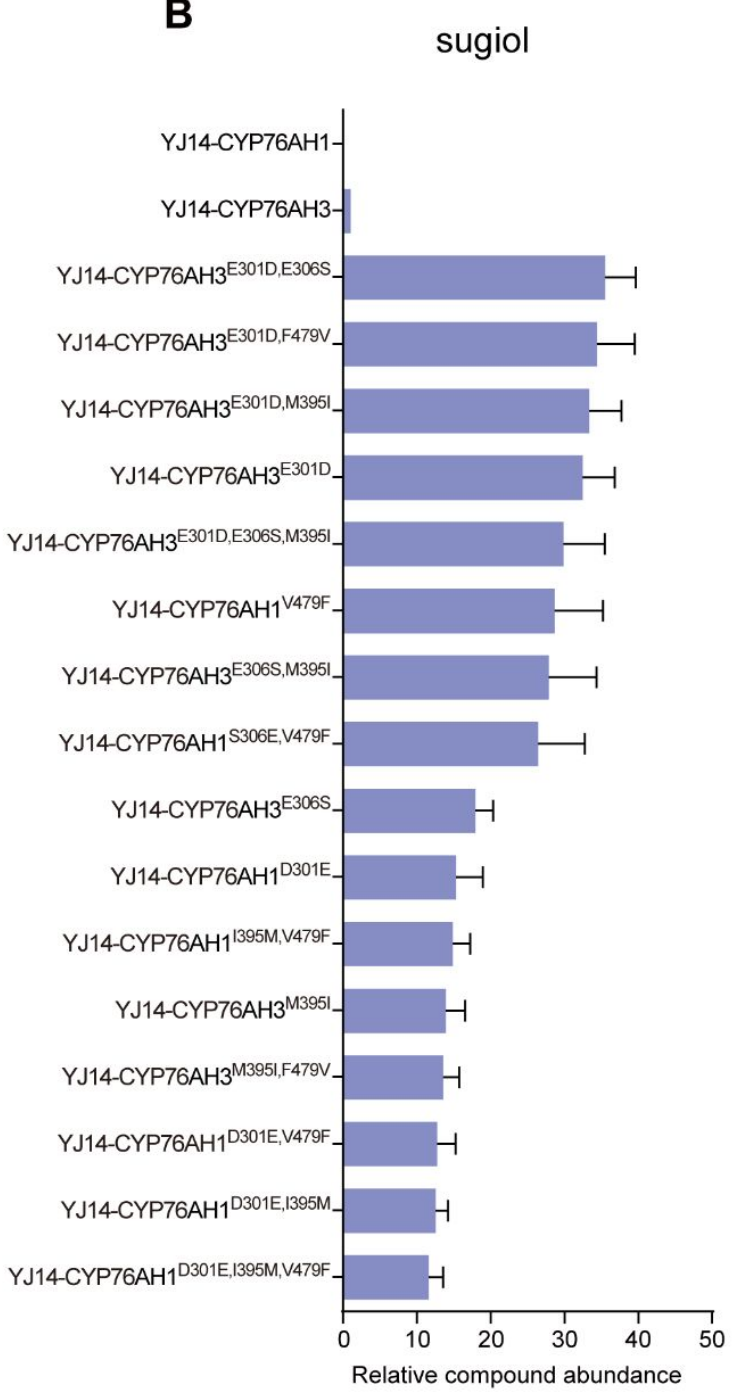

Figure S4. Relative yields of 11-hydroxy ferruginol(A), sugiol(B), and 11-hydroxy sugiol(C) from miltiradiene in yeast strains harboring SmCPR1 plus either CYP76AH1, CYP76AH3, and their mutated substitutes after fermentation. Yields higher than YJ14CYP76AH1/CYP76AH3 were showed. 
Table S1. S. cerevisiae strains used in this study.

\begin{tabular}{|c|c|c|}
\hline Strains & Genotype or characteristic & Resource \\
\hline BY4741 & MATa; his $3 \Delta 1$; leu $2 \Delta 0 ;$ met $15 \Delta 0 ;$ ura $3 \Delta 0$ & ATCC \\
\hline YJ14 & BY4741/pYX212-(BTS1-ERG20)+(SmKSL-SmCPS) & 15 \\
\hline YJ14-CYP76AH1 & YJ14/pESC-HIS-CYP76AH1/pESC-LEU-SmCPR1 & This study \\
\hline YJ14-CYP76AH3 & YJ14/pESC-HIS-CYP76AH3/pESC-LEU-SmCPR1 & This study \\
\hline YJ14-CYP76AH1 D301E,V479F & YJ14/pESC-HIS-CYP76AH1 ${ }^{D 301 E, V 479 F} / \mathrm{pESC}-\mathrm{LEU}-S m C P R 1$ & This study \\
\hline YJ51 & $\begin{array}{l}\text { BY4741/LEU2/MET15/pYX212-(BTS1-ERG20)+(SmKSL1- } \\
\text { SmCPS1)+CYP76AH1+SmCPR1/pESC-HIS-CYP76AH3 }\end{array}$ & 10 \\
\hline
\end{tabular}


Table S2. Primers used in this study.

\begin{tabular}{|c|c|}
\hline Primer & Sequence(5'to3') \\
\hline 76AH1-HisBamHI-F1 & CTTTAACGTCAAGGAGAAAAAACCCCGATGGATTCTTTTCCTCTCCTCGCCGCGCTCT \\
\hline 76AH1-D301E-R1 & CATGGCCCACTCGATCGAGGTCGTGTTCGTCTCCGATCCTCCCACGAACAAGTCCAGC \\
\hline 76AH1-D301E-F2 & GCTGGACTTGTTCGTGGGAGGATCGGAGACGAACACGACCTCGATCGAGTGGGCCATG \\
\hline 76AH1-HisBamHI-R2 & GACGCCCGGGCCCTATAGTGAGTCGTATTACGGCTAAGACTTAACTATTGGGATAATC \\
\hline 76AH1-HisBamHI-F1 & CTTTAACGTCAAGGAGAAAAAACCCCGATGGATTCTTTTCCTCTCCTCGCCGCGCTCT \\
\hline 76AH1-S306E-R1 & CATGGCCCACTCGATCTCGGTCGTGTTCGTGTCCGATCCTCCCACGAACAAGTCCAGC \\
\hline 76AH1-S306E-F2 & GCTGGACTTGTTCGTGGGAGGATCGGACACGAACACGACCGAGATCGAGTGGGCCATG \\
\hline 76AH1-HisBamHI-R2 & GACGCCCGGGCCCTATAGTGAGTCGTATTACGGCTAAGACTTAACTATTGGGATAATC \\
\hline 76AH1-HisBamHI-F1 & CTTTAACGTCAAGGAGAAAAAACCCCGATGGATTCTTTTCCTCTCCTCGCCGCGCTCT \\
\hline 76AH1-I395M-R1 & GGTCCAGATACTCGGATCCCTCCCCATCGCATACGCGTTGATGAGTATCTGAGTTCCC \\
\hline 76AH1-I395M-F2 & GGGAACTCAGATACTCATCAACGCGTATGCGATGGGGAGGGATCCGAGTATCTGGACC \\
\hline 76AH1-HisBamHI-R2 & GACGCCCGGGCCCTATAGTGAGTCGTATTACGGCTAAGACTTAACTATTGGGATAATC \\
\hline 76AH1-HisBamHI-F1 & СTTTAACGTCAAGGAGAAAAAACCCCGATGGATTCTTTTCCTCTCCTCGCCGCGCTCT \\
\hline 76AH1-V479F-R1 & GAACCCAAACAACTCGCCGGCGTGATCGGCGGCCGCCGTGGAGTCATCTTCCAGTTTC \\
\hline 76AH1-V479F-F2 & GAAACTGGAAGATGACTCCACGGCGGCCGCCGATCACGCCGGCGAGTTGTTTGGGTTC \\
\hline 76AH1-HisBamHI-R2 & GACGCCCGGGCCCTATAGTGAGTCGTATTACGGCTAAGACTTAACTATTGGGATAATC \\
\hline 76AH3-HisBamHI-F1 & СТTTAACGTCAAGGAGAAAAAACCCCGATGGATTCTTTCTCTCTTCTGGCTGCACTCT \\
\hline 76AH3-E301D-R1 & CATGATCCACTCGATCTCGGTGGTGCTCGTGTCCGAACCCCCGACGAAGAGGTCGAGC \\
\hline 76AH3-E301D-F2 & GCTCGACCTCTTCGTCGGGGGTTCGGACACGAGCACCACCGAGATCGAGTGGATCATG \\
\hline 76AH3-HisBamHI-R2 & GTGCCGCTCAGGATCGTTCCGTATAAGGCATGAGCTAAGACTTAACTATTGGGATAAT \\
\hline 76AH3-HisBamHI-F1 & CTTTAACGTCAAGGAGAAAAAACCCCGATGGATTCTTTCTCTCTTCTGGCTGCACTCT \\
\hline 76AH3-E306S-R1 & CATGATCCACTCGATCGAGGTGGTGCTCGTCTCCGAACCCCCGACGAAGAGGTCGAGC \\
\hline 76AH3-E306S-F2 & GCTCGACCTCTTCGTCGGGGGTTCGGAGACGAGCACCACCTCGATCGAGTGGATCATG \\
\hline 76AH3-HisBamHI-R4 & GTGCCGCTCAGGATCGTTCCGTATAAGGCATGAGCTAAGACTTAACTATTGGGATAAT \\
\hline 76AH3-HisBamHI-F1 & TCAAGGAGAAAAAACCCCGATGGATTCTTTCTCTCTTCTGGCT \\
\hline 76AH3-M395I-R1 & GTGGAGTCTCTGCCTATGGCCCACGCGTTGATGAGCACCTGAGT \\
\hline 76АH3-M395I-F2 & GACTCAGGTGCTCATCAACGCGTGGGCCATAGGCAGAGACTCCA \\
\hline 76AH3-HisBamHI-R2 & CTATAGTGAGTCGTATTACGGTGCCTTATACGGAACGATCC \\
\hline 76AH3-HisBamHI-F1 & СTTTAACGTCAAGGAGAAAAAACCCCGATGGATTCTTTCTCTCTTCTGGCTGCACTCT \\
\hline 76AH3-F479V-R1 & CACGCCAAACAACACGCCCTGGTGCTCGGCGAGGGGCGCATCGGGGCGTTCGAGCTTC \\
\hline 76AH3-F479V-F2 & GAAGCTCGAACGCCCCGATGCGCCCCTCGCCGAGCACCAGGGCGTGTTGTTTGGCGTG \\
\hline 76AH3-HisBamHI-R2 & GTGCCGCTCAGGATCGTTCCGTATAAGGCATGAGCTAAGACTTAACTATTGGGATAAT \\
\hline 76AH1-HisBamHI-F1 & СТTTAACGTCAAGGAGAAAAAACCCCGATGGATTCTTTTCCTCTCCTCGCCGCGCTCT \\
\hline 76AH1-D301E,S306E-R1 & CATGGCCCACTCGATCTCGGTCGTGTTCGTCTCCGATCCTCCCACGAACAAGTCCAGC \\
\hline 76AH1-D301E,S306E-F2 & GCTGGACTTGTTCGTGGGAGGATCGGAGACGAACACGACCGAGATCGAGTGGGCCATG \\
\hline 76AH1-HisBamHI-R2 & GACGCCCGGGCCCTATAGTGAGTCGTATTACGGCTAAGACTTAACTATTGGGATAATC \\
\hline 76AH1-HisBamHI-F1 & СTTTAACGTCAAGGAGAAAAAACCCCGATGGATTCTTTTCCTCTCCTCGCCGCGCTCT \\
\hline 76AH1-D301E-R1 & CATGGCCCACTCGATCGAGGTCGTGTTCGTCTCCGATCCTCCCACGAACAAGTCCAGC \\
\hline 76AH1-D301E-F2 & GCTGGACTTGTTCGTGGGAGGATCGGAGACGAACACGACCTCGATCGAGTGGGCCATG \\
\hline 76AH1-I395M-R2 & GGTCCAGATACTCGGATCCCTCCCCATCGCATACGCGTTGATGAGTATCTGAGTTCCC \\
\hline
\end{tabular}




\begin{tabular}{|c|c|}
\hline 76AH1-I395M-F3 & GGGAACTCAGATACTCATCAACGCGTATGCGATGGGGAGGGATCCGAGTATCTGGACC \\
\hline 76AH1-HisBamHI-R3 & GACGCCCGGGCCCTATAGTGAGTCGTATTACGGCTAAGACTTAACTATTGGGATAATC \\
\hline 76AH1-HisBamHI-F1 & CTTTAACGTCAAGGAGAAAAAACCCCGATGGATTCTTTTCCTCTCCTCGCCGCGCTCT \\
\hline 76AH1-D301E-R1 & CATGGCCCACTCGATCGAGGTCGTGTTCGTCTCCGATCCTCCCACGAACAAGTCCAGC \\
\hline 76AH1-D301E-F2 & GCTGGACTTGTTCGTGGGAGGATCGGAGACGAACACGACCTCGATCGAGTGGGCCATG \\
\hline 76AH1-V479F-R2 & GAACCCAAACAACTCGCCGGCGTGATCGGCGGCCGCCGTGGAGTCATCTTCCAGTTTC \\
\hline 76AH1-V479F-F3 & GAAACTGGAAGATGACTCCACGGCGGCCGCCGATCACGCCGGCGAGTTGTTTGGGTTC \\
\hline 76AH1-HisBamHI-R3 & GACGCCCGGGCCCTATAGTGAGTCGTATTACGGCTAAGACTTAACTATTGGGATAATC \\
\hline 76AH1-HisBamHI-F1 & CTTTAACGTCAAGGAGAAAAAACCCCGATGGATTCTTTTCCTCTCCTCGCCGCGCTCT \\
\hline 76AH1-S306E-R1 & CATGGCCCACTCGATCTCGGTCGTGTTCGTGTCCGATCCTCCCACGAACAAGTCCAGC \\
\hline 76AH1-S306E-F2 & GCTGGACTTGTTCGTGGGAGGATCGGACACGAACACGACCGAGATCGAGTGGGCCATG \\
\hline 76AH1-I395M-R2 & GGTCCAGATACTCGGATCCCTCCCCATCGCATACGCGTTGATGAGTATCTGAGTTCCC \\
\hline 76AH1-I395M-F3 & GGGAACTCAGATACTCATCAACGCGTATGCGATGGGGAGGGATCCGAGTATCTGGACC \\
\hline 76AH1-HisBamHI-R3 & GACGCCCGGGCCCTATAGTGAGTCGTATTACGGCTAAGACTTAACTATTGGGATAATC \\
\hline 76AH1-HisBamHI-F1 & CTTTAACGTCAAGGAGAAAAAACCCCGATGGATTCTTTTCCTCTCCTCGCCGCGCTCT \\
\hline 76AH1-S306E-R1 & CATGGCCCACTCGATCTCGGTCGTGTTCGTGTCCGATCCTCCCACGAACAAGTCCAGC \\
\hline 76AH1-S306E-F2 & GCTGGACTTGTTCGTGGGAGGATCGGACACGAACACGACCGAGATCGAGTGGGCCATG \\
\hline 76AH1-V479F-R2 & GAACCCAAACAACTCGCCGGCGTGATCGGCGGCCGCCGTGGAGTCATCTTCCAGTTTC \\
\hline 76AH1-V479F-F3 & GAAACTGGAAGATGACTCCACGGCGGCCGCCGATCACGCCGGCGAGTTGTTTGGGTTC \\
\hline 76AH1-HisBamHI-R3 & GACGCCCGGGCCCTATAGTGAGTCGTATTACGGCTAAGACTTAACTATTGGGATAATC \\
\hline 76AH1-HisBamHI-F1 & CTTTAACGTCAAGGAGAAAAAACCCCGATGGATTCTTTTCCTCTCCTCGCCGCGCTCT \\
\hline 76AH1-I395M-R1 & GGTCCAGATACTCGGATCCCTCCCCATCGCATACGCGTTGATGAGTATCTGAGTTCCC \\
\hline 76AH1-I395M-F2 & GGGAACTCAGATACTCATCAACGCGTATGCGATGGGGAGGGATCCGAGTATCTGGACC \\
\hline 76AH1-V479F-R2 & GAACCCAAACAACTCGCCGGCGTGATCGGCGGCCGCCGTGGAGTCATCTTCCAGTTTC \\
\hline 76AH1-V479F-F3 & GAAACTGGAAGATGACTCCACGGCGGCCGCCGATCACGCCGGCGAGTTGTTTGGGTTC \\
\hline 76AH1-HisBamHI-R3 & GACGCCCGGGCCCTATAGTGAGTCGTATTACGGCTAAGACTTAACTATTGGGATAATC \\
\hline 76AH3-HisBamHI-F1 & CTTTAACGTCAAGGAGAAAAAACCCCGATGGATTCTTTCTCTCTTCTGGCTGCACTCT \\
\hline 76AH3-E301D,E306S-R1 & CATGATCCACTCGATCGAGGTGGTGCTCGTGTCCGAACCCCCGACGAAGAGGTCGAGC \\
\hline 76AH3-E301D,E306S-F2 & GCTCGACCTCTTCGTCGGGGGTTCGGACACGAGCACCACCTCGATCGAGTGGATCATG \\
\hline 76AH3-HisBamHI-R2 & GTGCCGCTCAGGATCGTTCCGTATAAGGCATGAGCTAAGACTTAACTATTGGGATAAT \\
\hline 76AH3-HisBamHI-F1 & CTTTAACGTCAAGGAGAAAAAACCCCGATGGATTCTTTCTCTCTTCTGGCTGCACTCT \\
\hline 76AH3-E301D-R1 & CATGATCCACTCGATCTCGGTGGTGCTCGTGTCCGAACCCCCGACGAAGAGGTCGAGC \\
\hline 76AH3-E301D-F2 & GCTCGACCTCTTCGTCGGGGGTTCGGACACGAGCACCACCGAGATCGAGTGGATCATG \\
\hline 76АH3-M395I-R2 & CCAGATGGTGGAGTCTCTGCCTATGGCCCACGCGTTGATGAGCACCTGAGTCCCTTTC \\
\hline 76AH3-M395I-F3 & GAAAGGGACTCAGGTGCTCATCAACGCGTGGGCCATAGGCAGAGACTCCACCATCTGG \\
\hline 76AH3-HisBamHI-R3 & GTGCCGCTCAGGATCGTTCCGTATAAGGCATGAGCTAAGACTTAACTATTGGGATAAT \\
\hline 76AH3-HisBamHI-F1 & CTTTAACGTCAAGGAGAAAAAACCCCGATGGATTCTTTCTCTCTTCTGGCTGCACTCT \\
\hline 76AH3-E301D-R1 & CATGATCCACTCGATCTCGGTGGTGCTCGTGTCCGAACCCCCGACGAAGAGGTCGAGC \\
\hline 76AH3-E301D-F2 & GCTCGACCTCTTCGTCGGGGGTTCGGACACGAGCACCACCGAGATCGAGTGGATCATG \\
\hline 76AH3-F479V-R2 & CACGCCAAACAACACGCCCTGGTGCTCGGCGAGGGGCGCATCGGGGCGTTCGAGCTTC \\
\hline 76AH3-F479V-F3 & GAAGCTCGAACGCCCCGATGCGCCCCTCGCCGAGCACCAGGGCGTGTTGTTTGGCGTG \\
\hline
\end{tabular}




\begin{tabular}{|c|c|}
\hline 76AH3-HisBamHI-R3 & GTGCCGCTCAGGATCGTTCCGTATAAGGCATGAGCTAAGACTTAACTATTGGGATAAT \\
\hline 76AH3-HisBamHI-F1 & CTTTAACGTCAAGGAGAAAAAACCCCGATGGATTCTTTCTCTCTTCTGGCTGCACTCT \\
\hline 76AH3-E306S-R1 & CATGATCCACTCGATCGAGGTGGTGCTCGTCTCCGAACCCCCGACGAAGAGGTCGAGC \\
\hline 76AH3-E306S-F2 & GCTCGACCTCTTCGTCGGGGGTTCGGAGACGAGCACCACCTCGATCGAGTGGATCATG \\
\hline 76АH3-M395I-R2 & CCAGATGGTGGAGTCTCTGCCTATGGCCCACGCGTTGATGAGCACCTGAGTCCCTTTC \\
\hline 76АH3-M395I-F3 & GAAAGGGACTCAGGTGCTCATCAACGCGTGGGCCATAGGCAGAGACTCCACCATCTGG \\
\hline 76AH3-HisBamHI-R3 & GTGCCGCTCAGGATCGTTCCGTATAAGGCATGAGCTAAGACTTAACTATTGGGATAAT \\
\hline 76AH3-HisBamHI-F1 & CTTTAACGTCAAGGAGAAAAAACCCCGATGGATTCTTTCTCTCTTCTGGCTGCACTCT \\
\hline 76AH3-E306S-R1 & CATGATCCACTCGATCGAGGTGGTGCTCGTCTCCGAACCCCCGACGAAGAGGTCGAGC \\
\hline 76AH3-E306S-F2 & GCTCGACCTCTTCGTCGGGGGTTCGGAGACGAGCACCACCTCGATCGAGTGGATCATG \\
\hline 76AH3-F479V-R3 & CACGCCAAACAACACGCCCTGGTGCTCGGCGAGGGGCGCATCGGGGCGTTCGAGCTTC \\
\hline 76AH3-F479V-F3 & GAAGCTCGAACGCCCCGATGCGCCCCTCGCCGAGCACCAGGGCGTGTTGTTTGGCGTG \\
\hline 76AH3-HisBamHI-R3 & GTGCCGCTCAGGATCGTTCCGTATAAGGCATGAGCTAAGACTTAACTATTGGGATAAT \\
\hline 76AH3-HisBamHI-F1 & CTTTAACGTCAAGGAGAAAAAACCCCGATGGATTCTTTCTCTCTTCTGGCTGCACTCT \\
\hline 76АH3-M395I-R1 & CCAGATGGTGGAGTCTCTGCCTATGGCCCACGCGTTGATGAGCACCTGAGTCCCTTTC \\
\hline 76АH3-M395I-F2 & GAAAGGGACTCAGGTGCTCATCAACGCGTGGGCCATAGGCAGAGACTCCACCATCTGG \\
\hline 76AH3-F479V-R3 & CACGCCAAACAACACGCCCTGGTGCTCGGCGAGGGGCGCATCGGGGCGTTCGAGCTTC \\
\hline 76AH3-F479V-F3 & GAAGCTCGAACGCCCCGATGCGCCCCTCGCCGAGCACCAGGGCGTGTTGTTTGGCGTG \\
\hline 76AH3-HisBamHI-R3 & GTGCCGCTCAGGATCGTTCCGTATAAGGCATGAGCTAAGACTTAACTATTGGGATAAT \\
\hline 76AH1-HisBamHI-F1 & CTTTAACGTCAAGGAGAAAAAACCCCGATGGATTCTTTTCCTCTCCTCGCCGCGCTCT \\
\hline 76AH1-D301E,S306E-R1 & CATGGCCCACTCGATCTCGGTCGTGTTCGTCTCCGATCCTCCCACGAACAAGTCCAGC \\
\hline 76AH1-D301E,S306E-F2 & GCTGGACTTGTTCGTGGGAGGATCGGAGACGAACACGACCGAGATCGAGTGGGCCATG \\
\hline 76AH1-I395M-R2 & GGTCCAGATACTCGGATCCCTCCCCATCGCATACGCGTTGATGAGTATCTGAGTTCCC \\
\hline 76AH1-I395M-F3 & GGGAACTCAGATACTCATCAACGCGTATGCGATGGGGAGGGATCCGAGTATCTGGACC \\
\hline 76AH1-HisBamHI-R3 & GACGCCCGGGCCCTATAGTGAGTCGTATTACGGCTAAGACTTAACTATTGGGATAATC \\
\hline 76AH1-HisBamHI-F1 & CTTTAACGTCAAGGAGAAAAAACCCCGATGGATTCTTTTCCTCTCCTCGCCGCGCTCT \\
\hline 76AH1-D301E,S306E-R1 & CATGGCCCACTCGATCTCGGTCGTGTTCGTCTCCGATCCTCCCACGAACAAGTCCAGC \\
\hline 76AH1-D301E,S306E-F2 & GCTGGACTTGTTCGTGGGAGGATCGGAGACGAACACGACCGAGATCGAGTGGGCCATG \\
\hline 76AH1-V479F-R2 & GAACCCAAACAACTCGCCGGCGTGATCGGCGGCCGCCGTGGAGTCATCTTCCAGTTTC \\
\hline 76AH1-V479F-F3 & GAAACTGGAAGATGACTCCACGGCGGCCGCCGATCACGCCGGCGAGTTGTTTGGGTTC \\
\hline 76AH1-HisBamHI-R3 & GACGCCCGGGCCCTATAGTGAGTCGTATTACGGCTAAGACTTAACTATTGGGATAATC \\
\hline 76AH1-HisBamHI-F1 & CTTTAACGTCAAGGAGAAAAAACCCCGATGGATTCTTTTCCTCTCCTCGCCGCGCTCT \\
\hline 76AH1-D301E-R1 & CATGGCCCACTCGATCGAGGTCGTGTTCGTCTCCGATCCTCCCACGAACAAGTCCAGC \\
\hline 76AH1-D301E-F2 & GCTGGACTTGTTCGTGGGAGGATCGGAGACGAACACGACCTCGATCGAGTGGGCCATG \\
\hline 76AH1-I395M-R2 & GGTCCAGATACTCGGATCCCTCCCCATCGCATACGCGTTGATGAGTATCTGAGTTCCC \\
\hline 76AH1-I395M-F3 & GGGAACTCAGATACTCATCAACGCGTATGCGATGGGGAGGGATCCGAGTATCTGGACC \\
\hline 76AH1-V479F-R3 & GAACCCAAACAACTCGCCGGCGTGATCGGCGGCCGCCGTGGAGTCATCTTCCAGTTTC \\
\hline 76AH1-V479F-F4 & GAAACTGGAAGATGACTCCACGGCGGCCGCCGATCACGCCGGCGAGTTGTTTGGGTTC \\
\hline 76AH1-HisBamHI-R4 & GACGCCCGGGCCCTATAGTGAGTCGTATTACGGCTAAGACTTAACTATTGGGATAATC \\
\hline 76AH1-HisBamHI-F1 & CTTTAACGTCAAGGAGAAAAAACCCCGATGGATTCTTTTCCTCTCCTCGCCGCGCTCT \\
\hline 76AH1-S306E-R1 & CATGGCCCACTCGATCTCGGTCGTGTTCGTGTCCGATCCTCCCACGAACAAGTCCAGC \\
\hline
\end{tabular}




\begin{tabular}{|c|c|}
\hline 76AH1-S306E-F2 & GCTGGACTTGTTCGTGGGAGGATCGGACACGAACACGACCGAGATCGAGTGGGCCATG \\
\hline 76AH1-I395M-R2 & GGTCCAGATACTCGGATCCCTCCCCATCGCATACGCGTTGATGAGTATCTGAGTTCCC \\
\hline 76AH1-I395M-F3 & GGGAACTCAGATACTCATCAACGCGTATGCGATGGGGAGGGATCCGAGTATCTGGACC \\
\hline 76AH1-V479F-R3 & GAACCCAAACAACTCGCCGGCGTGATCGGCGGCCGCCGTGGAGTCATCTTCCAGTTTC \\
\hline 76AH1-V479F-F4 & GAAACTGGAAGATGACTCCACGGCGGCCGCCGATCACGCCGGCGAGTTGTTTGGGTTC \\
\hline 76AH1-HisBamHI-R4 & GACGCCCGGGCCCTATAGTGAGTCGTATTACGGCTAAGACTTAACTATTGGGATAATC \\
\hline 76AH3-HisBamHI-F1 & CTTTAACGTCAAGGAGAAAAAACCCCGATGGATTCTTTCTCTCTTCTGGCTGCACTCT \\
\hline 76AH3-E301D,E306S-R1 & CATGATCCACTCGATCGAGGTGGTGCTCGTGTCCGAACCCCCGACGAAGAGGTCGAGC \\
\hline 76AH3-E301D,E306S-F2 & GCTCGACCTCTTCGTCGGGGGTTCGGACACGAGCACCACCTCGATCGAGTGGATCATG \\
\hline 76AH3-M395I-R2 & CCAGATGGTGGAGTCTCTGCCTATGGCCCACGCGTTGATGAGCACCTGAGTCCCTTTC \\
\hline 76AH3-M395I-F3 & GAAAGGGACTCAGGTGCTCATCAACGCGTGGGCCATAGGCAGAGACTCCACCATCTGG \\
\hline 76AH3-HisBamHI-R3 & GTGCCGCTCAGGATCGTTCCGTATAAGGCATGAGCTAAGACTTAACTATTGGGATAAT \\
\hline 76AH3-HisBamHI-F1 & CTTTAACGTCAAGGAGAAAAAACCCCGATGGATTCTTTCTCTCTTCTGGCTGCACTCT \\
\hline 76AH3-E301D,E306S-R1 & CATGATCCACTCGATCGAGGTGGTGCTCGTGTCCGAACCCCCGACGAAGAGGTCGAGC \\
\hline 76AH3-E301D,E306S-F2 & GCTCGACCTCTTCGTCGGGGGTTCGGACACGAGCACCACCTCGATCGAGTGGATCATG \\
\hline 76AH3-F479V-R3 & CACGCCAAACAACACGCCCTGGTGCTCGGCGAGGGGCGCATCGGGGCGTTCGAGCTTC \\
\hline 76AH3-F479V-F3 & GAAGCTCGAACGCCCCGATGCGCCCCTCGCCGAGCACCAGGGCGTGTTGTTTGGCGTG \\
\hline 76AH3-HisBamHI-R3 & GTGCCGCTCAGGATCGTTCCGTATAAGGCATGAGCTAAGACTTAACTATTGGGATAAT \\
\hline 76AH3-HisBamHI-F1 & CTTTAACGTCAAGGAGAAAAAACCCCGATGGATTCTTTCTCTCTTCTGGCTGCACTCT \\
\hline 76AH3-E301D-R1 & CATGATCCACTCGATCTCGGTGGTGCTCGTGTCCGAACCCCCGACGAAGAGGTCGAGC \\
\hline 76AH3-E301D-F2 & GCTCGACCTCTTCGTCGGGGGTTCGGACACGAGCACCACCGAGATCGAGTGGATCATG \\
\hline 76AH3-M395I-R2 & CCAGATGGTGGAGTCTCTGCCTATGGCCCACGCGTTGATGAGCACCTGAGTCCCTTTC \\
\hline 76AH3-M395I-F3 & GAAAGGGACTCAGGTGCTCATCAACGCGTGGGCCATAGGCAGAGACTCCACCATCTGG \\
\hline 76AH3-F479V-R3 & CACGCCAAACAACACGCCCTGGTGCTCGGCGAGGGGCGCATCGGGGCGTTCGAGCTTC \\
\hline 76AH3-F479V-F3 & GAAGCTCGAACGCCCCGATGCGCCCCTCGCCGAGCACCAGGGCGTGTTGTTTGGCGTG \\
\hline 76AH3-HisBamHI-R4 & GTGCCGCTCAGGATCGTTCCGTATAAGGCATGAGCTAAGACTTAACTATTGGGATAAT \\
\hline 76AH3-HisBamHI-F1 & CTTTAACGTCAAGGAGAAAAAACCCCGATGGATTCTTTCTCTCTTCTGGCTGCACTCT \\
\hline 76AH3-E306S-R1 & CATGATCCACTCGATCGAGGTGGTGCTCGTCTCCGAACCCCCGACGAAGAGGTCGAGC \\
\hline 76AH3-E306S-F2 & GCTCGACCTCTTCGTCGGGGGTTCGGAGACGAGCACCACCTCGATCGAGTGGATCATG \\
\hline 76AH3-M395I-R2 & CCAGATGGTGGAGTCTCTGCCTATGGCCCACGCGTTGATGAGCACCTGAGTCCCTTTC \\
\hline 76AH3-M395I-F3 & GAAAGGGACTCAGGTGCTCATCAACGCGTGGGCCATAGGCAGAGACTCCACCATCTGG \\
\hline 76AH3-F479V-R3 & CACGCCAAACAACACGCCCTGGTGCTCGGCGAGGGGCGCATCGGGGCGTTCGAGCTTC \\
\hline 76AH3-F479V-F3 & GAAGCTCGAACGCCCCGATGCGCCCCTCGCCGAGCACCAGGGCGTGTTGTTTGGCGTG \\
\hline 76AH3-HisBamHI-R4 & GTGCCGCTCAGGATCGTTCCGTATAAGGCATGAGCTAAGACTTAACTATTGGGATAAT \\
\hline 76AH1-HisBamHI-F1 & CTTTAACGTCAAGGAGAAAAAACCCCGATGGATTCTTTTCCTCTCCTCGCCGCGCTCT \\
\hline 76AH1-D301E,S306E-R1 & CATGGCCCACTCGATCTCGGTCGTGTTCGTCTCCGATCCTCCCACGAACAAGTCCAGC \\
\hline 76AH1-D301E,S306E-F2 & GCTGGACTTGTTCGTGGGAGGATCGGAGACGAACACGACCGAGATCGAGTGGGCCATG \\
\hline 76AH1-I395M-R2 & GGTCCAGATACTCGGATCCCTCCCCATCGCATACGCGTTGATGAGTATCTGAGTTCCC \\
\hline 76AH1-I395M-F3 & GGGAACTCAGATACTCATCAACGCGTATGCGATGGGGAGGGATCCGAGTATCTGGACC \\
\hline 76AH1-V479F-R3 & GAACCCAAACAACTCGCCGGCGTGATCGGCGGCCGCCGTGGAGTCATCTTCCAGTTTC \\
\hline 76AH1-V479F-F4 & GAAACTGGAAGATGACTCCACGGCGGCCGCCGATCACGCCGGCGAGTTGTTTGGGTTC \\
\hline
\end{tabular}




\begin{tabular}{|l|l|}
\hline 76AH1-HisBamHI-R4 & GACGCCCGGGCCCTATAGTGAGTCGTATTACGGCTAAGACTTAACTATTGGGATAATC \\
\hline 76AH3-HisBamHI-F1 & CTTTAACGTCAAGGAGAAAAAACCCCGATGGATTCTTTCTCTCTTCTGGCTGCACTCT \\
\hline 76AH3-E301D,E306S-R1 & CATGATCCACTCGATCGAGGTGGTGCTCGTGTCCGAACCCCCGACGAAGAGGTCGAGC \\
\hline 76AH3-E301D,E306S-F2 & GCTCGACCTCTTCGTCGGGGGTTCGGACACGAGCACCACCTCGATCGAGTGGATCATG \\
\hline 76AH3-M395I-R2 & CCAGATGGTGGAGTCTCTGCCTATGGCCCACGCGTTGATGAGCACCTGAGTCCCTTTC \\
\hline 76AH3-M395I-F3 & GAAAGGGACTCAGGTGCTCATCAACGCGTGGGCCATAGGCAGAGACTCCACCATCTGG \\
\hline 76AH3-F479V-R3 & CACGCCAAACAACACGCCCTGGTGCTCGGCGAGGGGCGCATCGGGGCGTTCGAGCTTC \\
\hline 76AH3-F479V-F3 & GAAGCTCGAACGCCCCGATGCGCCCCTCGCCGAGCACCAGGGCGTGTTGTTTGGCGTG \\
\hline 76AH3-HisBamHI-R4 & GTGCCGCTCAGGATCGTTCCGTATAAGGCATGAGCTAAGACTTAACTATTGGGATAAT \\
\hline
\end{tabular}

